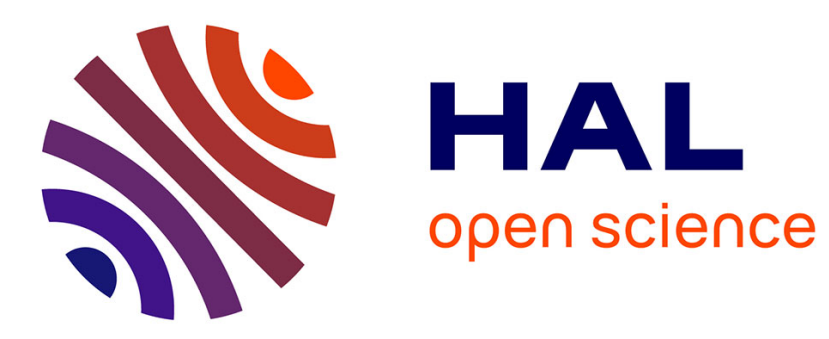

\title{
Micro-mechanics and continuum damage mechanics
}

\author{
Alain Burr, François Hild, F. A. Leckie
}

\section{To cite this version:}

Alain Burr, François Hild, F. A. Leckie. Micro-mechanics and continuum damage mechanics. Archive of Applied Mechanics, 1995, 65 (7), pp.437 - 456. 10.1007/BF00835656 . hal-01636227

\section{HAL Id: hal-01636227 \\ https://hal.science/hal-01636227}

Submitted on 31 Oct 2019

HAL is a multi-disciplinary open access archive for the deposit and dissemination of scientific research documents, whether they are published or not. The documents may come from teaching and research institutions in France or abroad, or from public or private research centers.
L'archive ouverte pluridisciplinaire HAL, est destinée au dépôt et à la diffusion de documents scientifiques de niveau recherche, publiés ou non, émanant des établissements d'enseignement et de recherche français ou étrangers, des laboratoires publics ou privés. 


\title{
Micro-mechanics and continuum damage mechanics
}

\author{
A. Burr, F. Hild, F. A. Leckie
}

Summary Continuum Damage Mechanics has been applied successfully to technical problems since the idea was introduced by Kachanov almost 40 years ago. In keeping with the traditions of mechanics, the formulation was based on the results of mechanical tests on specimens whose size is measured in centimeters. To model the observations which describe the deterioration of material properties it was found necessary to introduce internal variables referred to as 'damage'. The approach is phenomenological, with only a minimal attempt to provide a physical interpretation of damage. For this reason the approach has had little appeal to those whose interest is in the physical mechanisms which cause material deterioration. In this presentation a description is given of attempts to develop continuum damage mechanics so that the relationship with the physical mechanism approach is less abrupt. The procedure is illustrated with reference to ceramic matrix composites.

Key words continuum damage, micro-mechanics, ceramic matrix, composite modelling

1

\section{Introduction}

By contrast with fracture mechanics, which describes the behavior of a component weakened by the presence of a single well-defined crack, Continuum Damage Mechanics (CDM) attempts to deal with the circumstances when multiple cracking or voidage is evidently the dominant degradation and failure mechanism. The uniform distribution of voids and cracks, which was observed in high-temperature creep rupture, attracted the attention of Kachanov in 1958 [1], Kachanov was apparently the first to attempt a description of a creep rupture model by introducing an internal damage variable into the constitutive equations describing the creep behavior. While microscopic examinations clearly identified the presence of voids and cracks, the influence of damage and its growth were deduced from the results of the traditional tests performed by mechanical engineers, and given in the form

$\frac{\varepsilon}{\varepsilon_{0}}=\left(\frac{\sigma}{\sigma_{0}(1-D)}\right)^{n}$,

$\stackrel{\circ}{D}=\left(\frac{\sigma}{\sigma_{0}(1-D)}\right)^{p}$,

where $n, p, \varepsilon_{0}, \sigma_{0}$ were selected to fit experiments, $\sigma$ and $\varepsilon$ denote stress, and strain, respectively. The damage $D$ was assumed to be a scalar, in spite of contrary microscopic evidence which indicated the damage to have directional properties. Equations of this form have been used

Dr. A. Burr, Dr. F. Hild

Laboratoire de Mécanique et Technologie,

E.N.S. de Cachan/C.N.R.S./Université Paris 6, 61 avenue du Président

Wilson, F-94235 Cachan Cedex, France

Prof. F. A. Leckie

Department of Mechanical and Environmental Engineering,

University of California, Santa Barbara CA 93106-5070, USA

This work was supported by the Defence Advanced Research Project Agency through the University Research Initiative under Office of Naval Research Contract No. N-00014-92-J-1808. 
is referred to as the 'effective stress' [4]. The approach has been fully described and utilized by Lemaitre and co-workers [5]. The procedure has the advantage that it is simple and can be readily related to the results of macroscopic tests, and by simple generalization can provide a means of extending the effects of damage on more complex phenomena, such as anisothermal viscoplasticity [6], by measuring stiffness loss as an indication of damage accumulation [7].

The behavior of concrete is another example which lends itself well to a CDM description. In this case, when concrete is loaded, microcracks appear at the interface of the cementitious matrix and the aggregate. Using the concept of 'effective stress', Mazars [8] has proposed simple damage laws which have proved to be useful in predicting the behavior of complex engineering components such as dams. Other applications can be found in the work of Krajcinovic [2].

In the engineering development of CDM there is little attempt to give a precise physical description of damage, since the focus is on the prediction of component behavior for which the length scale is many times that associated with the physical damage in the material. By temperament and training, design engineers tend to place greater confidence in the results of appropriate mechanical tests than they do in the results of studies of physical mechanisms. However, the inability to respond to the inquiry, 'What is this damage $D$ ?', has limited the wider acceptance of the CDM approach and denied it the benefits to be gained from understanding the physical processes of damage growth. Ashby and Dyson [9] were able to establish the connection between the damage parameter $D$ and the physical deterioration occurring in metals operating at elevated temperatures. A major contribution of this study was to identify the operating condition within which any particular mechanism is operative. Subsequently, Cocks and Leckie [10] recast the results of the Ashby and Dyson study in the CDM format. Using this approach, Hall and Hayhurst [11] demonstrated how CDM procedures can predict with precision the type and growth of damage occurring in complex engineering components.

Recently, the authors were involved in a study of the properties of Ceramic Matrix Composites (CMCs) consisting of a brittle matrix reinforced by continuous fibers and which, when loaded, exhibit multiple matrix cracking. The matrix cracking is known to be accompanied by slipping at the fiber/matrix interface which results in irreversible macroscopic strains. Following recent materials science studies [12], substantial knowledge exists about the damage mechanisms occurring in these materials, and the effect they have on material properties. These circumstances provided an opportunity to develop a CDM description for CMCs based on the results of materials science, which is the subject of this presentation. It is worthy of note that the CDM description was being developed at a time when little material was available to support an extensive test program. These circumstances emphasized the need of an approach, which is sufficiently general to allow for systematic improvement as material science knowledge unfolded, and the results of further tests became available.

2

\section{The formulation of a physically-based COM model}

The general structure for the constitutive equations is obtained by following a thermodynamic approach [13-15] in identifying a finite number of internal state variables. The state of the material is then described by calculating the Helmholz free energy density $\psi$, from which the associated thermodynamic forces may be derived. Once the state variables and the associated forces are defined, the formulation is then completed by defining growth laws of the state variables in terms of their associated forces. It is common at this stage [3] to define the damage growth laws by the introduction of a potential of the internal forces. This approach is not used in the present development, and the growth laws are obtained directly from experimental results.

In addition to the total strain tensor, the variables defining the state of the CMC include the size, shape and distribution of the matrix cracks. The materials science studies also indicate [12] that matrix cracking is accompanied by slipping at the fiber/matrix interface, so that the degree of interface slipping must be included in the state variables. The state of the material can also be defined in a very compact form by a scalar which is the Helmholz free energy. The free energy is calculated by following any reversible path to the current state.

In the present study, the reversible path consists of two steps. The first step consists in introducing into the unloaded cracked material the interface slips by a series of cutting, displacing and resealing operations. This approach was introduced by Volterra [16], and used to analyze the elastic behavior of homogeneous and isotropic media by considering the elastic properties of a cut cylinder [16;17], as well as inclusions in an infinite medium [18], or to study creeping materials [10]. In each 
case, the contribution to the free energy density can be written as

$\psi^{s}=\psi^{s}\left(\beta_{k}, \alpha\right)$

where each $\beta_{k}$ represents an interface slip, and $\alpha$ the resulting macroscopic inelastic strain tensor.

The second step consists in loading the cracked material elastically with the elastic strain $\varepsilon^{e}$ so that the free energy is given by

$\psi^{e}=\frac{1}{2} \varepsilon^{e}: \mathbf{C}\left(\delta_{k}\right): \varepsilon^{e}$

where $\mathbf{C}$ is the elastic stiffness tensor which is a function of the crack sizes and distributions $\delta_{k}$, and $\varepsilon^{e}$ is the elastic strain tensor resulting from the application of load.

The total free energy density associated with the present state is given by summing the two energy components so that

$\psi=\frac{1}{2} \varepsilon^{e}: \mathbf{C}\left(\delta_{k}\right): \varepsilon^{e}+\psi^{s}\left(\beta_{k}, \boldsymbol{\alpha}\right)$,

where the usual summation convention is used, " $\because$ represents the contraction with respect to two indices. The strain partition $\varepsilon^{e}=\varepsilon-\alpha$, where $\varepsilon$ is the total strain tensor, permits the free energy density to be written as follows:

$\psi=\frac{1}{2}(\varepsilon-\alpha): C\left(\delta_{k}\right):(\varepsilon-\alpha)+\psi^{s}\left(\beta_{k}, \alpha\right)$

The thermodynamic forces associated with the state variables $\varepsilon, \alpha, \delta_{k}$ and $\beta_{k}$ (i.e. $\sigma, \mathrm{X}, G_{k}$ and $s_{k}$, respectively) can be found by differentiation of Eq. (7),

$\dot{\psi}=\sigma: \stackrel{\AA}{\varepsilon}+\mathbf{X}: \stackrel{\circ}{\mathrm{q}}+G_{k} \cdot \stackrel{\circ}{\delta}_{k}+s_{k} \cdot \stackrel{\circ}{\beta}_{k}$,

where

$\boldsymbol{\sigma}=\frac{\partial \psi}{\partial \boldsymbol{\varepsilon}}, \quad \mathbf{X}=\frac{\partial \psi}{\partial \boldsymbol{\alpha}}, \quad G_{k}=\frac{\partial \psi}{\partial \delta_{k}} \quad$ and $\quad s_{k}=\frac{\partial \psi}{\partial \beta_{k}}$

and where $s_{k}, G_{k}$ have the same tensorial nature as $\beta_{k}, \delta_{k}$ and $\cdot$ represents contraction with respect to the number of indices equal to the rank of the tensors $\beta_{k}$ and $\delta_{k}$.

The results obtained in this section are central to the developments in the remainder of this paper. In Sect. 3 the elastic properties resulting from matrix cracking shall be analyzed. The free energy associated with interface slip is discussed in Sect. 4 . The growth laws relating damage to the internal forces are discussed in Sects. 5 and 6. Instead of following the conventional procedure of identifying a scalar potential to define the growth laws as used in [13-15], a different approach is described which combines the results of micromechanics with the results of conventional mechanical tests.

3

\section{Elastic properties of isotropic and anisotropic damaged materials}

3.1 Isotropic description

The effect of matrix voids and cracks on the elastic properties of materials have received considerable attention. The continuum damage description of the coupling between elasticity and damage has been presented by Lemaitre and Marquis [19], and the micromechanics for specific distributions of cracks have been completed for example by Budiansky [20;21]. It is the expression for the Helmholz free energy density $\psi$ which is used to determine the associated internal forces. It proves to be simpler in some problems to use the Gibbs specific enthalpy $\varphi$ which is the Legendre transformation of the Helmholtz free energy density according to the relation

$\boldsymbol{\sigma}: \varepsilon=\psi+\varphi$ 
The Gibbs specific enthalpy $\varphi$ shall be introduced later in this section when appropriate.

For an isotropic material, the initial elastic properties are defined by the shear modulus $G$ and the bulk modulus $K$. If, after degradation, the material remains isotropic the elastic properties are defined by the new values of the shear modulus $\tilde{G}$ and bulk modulus $\tilde{K}$. The elastic energy density, which is here identical to the Helmholtz free energy density $\psi$, is written as follows:

$2 \psi=2 \tilde{\mathrm{G}} \mathrm{e}: \mathrm{e}+\tilde{K} \varepsilon_{v}^{2}$

where $\mathbf{e}$ is the deviatoric strain tensor, and $\varepsilon_{v}$ denotes the volumetric strain. The associated force to the infinitesimal strain tensor $\varepsilon$ is the Cauchy stress tensor $\sigma$

$\sigma=\frac{\partial \psi}{\partial \boldsymbol{\varepsilon}}$

and the associated force(s) to the damage variable(s) $D$ are obtained by partial differentiation of the state potential with respect to the corresponding internal variables. The exact expression depends on the details of the model, as shown in the following.

A first attempt within the context of CDM to write a state potential for isotropic damaged materials was the phenomenological description of Lemaitre and Chaboche [22]. The formulation is based on the assumption of 'equivalence of the deformations hypothesis' [23] that states that 'any strain constitutive law for a damaged material may be derived in the same way as for a virgin material except that the usual stress is replaced by the effective stress'. It is postulated that in the case of an isotropic material, only one damage variable $D$ is needed. The consequence is that the two elastic parameters are written as a function of one scalar damage variable $D$

$$
\begin{aligned}
& \frac{\tilde{G}}{G}=1-D, \\
& \frac{\tilde{K}}{K}=1-D .
\end{aligned}
$$

It is noted that with this definition, Poisson's ratio remains unaltered compared to the undamaged material. The associated force to the damage variable $D$ is referred to as the energy release rate density $Y[24]$, and is defined as

$$
Y=-\frac{\partial \psi}{\partial D}=\frac{1}{2}\left(2 G \mathrm{e}: \mathrm{e}+K \varepsilon_{v}^{2}\right)
$$

These associated forces (here $Y$ ) are usually used as driving forces of the internal state variables (here $D$ ).

Ladevèze [25] originally proposed a general expression of the Gibbs specific enthalpy as a function of two independent damage variables $d$ and $\delta$ in the so-called 'kinematics of zeroth order'. However, the same formalism can be used when the Helmholtz free energy density is analyzed, provided a partition of the strain tensor is written in terms of its deviatoric and spherical parts. The two elastic parameters are written as

$$
\begin{aligned}
& \frac{\tilde{G}}{G}=1-d, \\
& \frac{\tilde{K}}{K}=1-\delta .
\end{aligned}
$$

The two forces associated with the damage variables $d$ and $\delta$ are respectively given by

$$
\begin{aligned}
& Y_{d}=-\frac{\partial \psi}{\partial d}=G \mathrm{e}: \mathrm{e}, \\
& Y_{\delta}=-\frac{\partial \psi}{\partial \delta}=\frac{1}{2} K \varepsilon_{v^{*}}^{2}
\end{aligned}
$$

These first two models are completely phenomenological. On the other hand, the micromechanics analysis performed by Budiansky [20] predicts the effect of a dilute concentration of voids on 
the macroscopic elastic properties. The new values of the shear and bulk moduli resulting from an average void density $\bar{\omega}_{v}$ are given by

$\frac{\tilde{G}}{G}=1-15 \frac{1-v}{7-5 \nu} \bar{\omega}_{v}$,
$\frac{\tilde{K}}{K}=1-\frac{3(1-v)}{2(1-2 v)} \bar{\omega}_{v}$,

where $v$ is the Poisson's ratio of the undamaged material. This micromechanics approach gives results which are dependent on a single state variable similar to that defined by Lemaitre and Chaboche in Eq. (13-14). However, there are differences in the predictions of the two approaches as can be readily demonstrated by making the substitution

$D=15 \frac{1-v}{7-5 v} \bar{\omega}_{\nu}$

Then Eqs. (20-21) become

$\frac{\widetilde{G}}{G}=1-D$,

$\frac{\tilde{K}}{K}=1-\frac{9 K+8 G}{20 G} D$.

The corresponding thermodynamic force or energy release rate density is given by

$Y=-\frac{\partial \psi}{\partial D}=\frac{1}{2}\left(2 G \mathrm{e}: \mathrm{e}+\frac{9 K+8 G}{20 G} K \varepsilon_{\nu}^{2}\right)$

which is different from the expression given in Eq. (15).

Budiansky and $O^{\prime}$ Connel [21] have also performed similar calculations to determine the changes in the elastic properties of an isotropic distribution of penny-shaped cracks. The relationship between the shear and bulk moduli changes, and the average crack density $\bar{\omega}_{c}$ is given by

$\frac{\tilde{G}}{G}=1-\frac{32}{45} \frac{(1-\tilde{v})(5-\tilde{v})}{2-\tilde{v}} \bar{\omega}_{c}$

$\frac{\tilde{K}}{K}=1-\frac{16}{9} \frac{1-\tilde{v}^{2}}{1-2 \tilde{v}} \bar{\omega}_{c}$

with

$\bar{\omega}_{c}=\frac{45}{16} \frac{(v-\tilde{v})(2-\tilde{v})}{\left(1-\tilde{v}^{2}\right)[10 v-\tilde{v}(1+3 v)]}$,

where $\tilde{v}$ denotes the Poisson's ratio of the damaged material. Setting

$D=\frac{32}{45} \frac{(1-\tilde{v})(5-\tilde{v})}{2-\tilde{v}} \bar{\omega}_{c}$

shows that in this case there is no linear relationship between the damage variable $D$ and the average crack density $\bar{w}_{c^{\prime}}$ It is worth remembering that Eqs. (26-29) are valid when the crack is embedded in homogeneous equivalent material (i.e. using a self-consistent scheme [26]). When $v=0$, then Eqs. (26-27) are simplified and are identical with the results given in Eqs. (13-14) with

$D=\frac{16}{9} \bar{\omega}_{c}$.

The above results have been obtained for isotropic and homogeneous damage, and are unable to model the influence of randomly distributed damage. Using a pattern approach [27] applied to a generalized self-consistent scheme $[28 ; 29]$ it can be shown that the shear modulus of a material containing a distribution of voids is not only dependent upon the average concentration (i.e. the average 


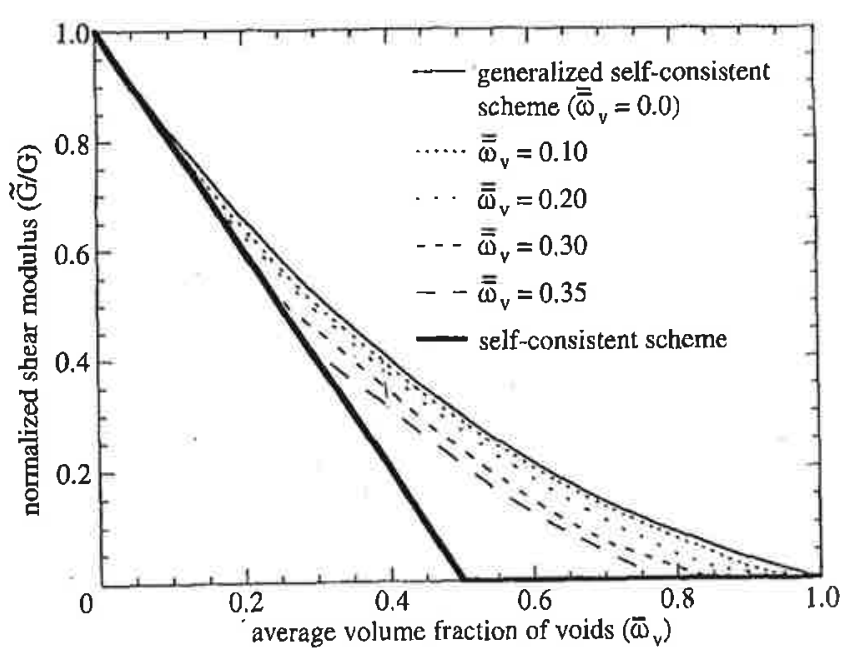

Fig. 1. Normalized shear modulus $\tilde{G} / G$ as a function of volume fraction of voids $\bar{\omega}_{v}$ and the corresponding standard deviation $\bar{\omega}_{v}$

void density $\left.\bar{\omega}_{v}\right)$ but also on the standard deviation $\left(\bar{\omega}_{v}\right)$, as shown in Fig. 1. Therefore, the damage variable $D_{v}=\tilde{G} / G$ at least depends upon the first two moments of the void distribution. Figure 1 also shows that it is possible to describe continuously the change from the result given by a self-consistent scheme to that given by a generalized self-consistent scheme, by varying the standard deviation of the distribution of voids.

When cracks and cavities are present, the simplest result to be derived is by assuming no interaction between the two defect populations as well as a Taylor scheme. The overall behavior is still isotropic, but now it depends on the statistical distributions of the two populations. From the previous results, the average free energy density $\psi$ is then related to the average free energy density $\psi_{c}$ of a cracked system, and the average free energy density $\psi_{\nu}$ of a system containing voids by

$\psi=(1-f) \psi_{c}\left(\bar{\omega}_{c}\right)+f \psi_{v}\left(\bar{\omega}_{v}\right)$

so that

$\tilde{G}\left(\bar{\omega}_{c}, \bar{\omega}_{v}, v\right)=(1-f) \tilde{G}_{c}\left(\bar{\omega}_{c}, v\right)+f \tilde{G}_{v}\left(\bar{\omega}_{v}, v\right)=G(1-d)$,
$\tilde{K}\left(\bar{\omega}_{c}, \bar{\omega}_{v}, v\right)=(1-f) \tilde{K}_{c}\left(\bar{\omega}_{c}, v\right)+f \tilde{K}_{v}\left(\bar{\omega}_{v}, v\right)=K(1-d)$,

where $f$ is the volume proportion of material embedding voids. The average void density and the average crack density are the two independent damage variables required to model the behavior. The forces associated with the average crack density $\bar{\omega}_{c}$ and the average void density $\bar{\omega}_{\nu}$ are defined by

$$
\begin{aligned}
& Y_{c}=-\frac{\partial \psi}{\partial \bar{\omega}_{c}}=\frac{1}{2}(1-f)\left(2 \frac{\partial \tilde{G}_{c}\left(\bar{\omega}_{c}, v\right)}{\partial \bar{\omega}_{c}} \mathbf{e}: \mathbf{e}+\frac{\partial \tilde{K}_{c}\left(\bar{\omega}_{c}, v\right)}{\partial \bar{\omega}_{c}} \varepsilon_{v}^{2}\right), \\
& Y_{v}=-\frac{\partial \psi}{\partial \bar{\omega}_{v}}=\frac{1}{2} f\left(2 \frac{\partial \tilde{G}_{v}\left(\bar{\omega}_{v}, v\right)}{\partial \bar{\omega}_{v}} \mathbf{e}: \mathbf{e}+\frac{\partial \tilde{K}_{v}\left(\bar{\omega}_{v}, v\right)}{\partial \bar{\omega}_{v}} \varepsilon_{v}^{2}\right) .
\end{aligned}
$$

If the internal variables selected are the damage variables $d$ and $\delta$, then the associated $Y_{d}$ and $Y_{\delta}$ defined in Eqs. $(18-19)$ are functions of the average crack density as well as the void density. From this it can be appreciated that the formulation of the free energy from micromechanics has the advantage that it allows more physical insight.

Even though the initial behavior of a damaged material may be isotropic, loading conditions shall result in an evolution of cracks which introduce anisotropic behavior. For example, this is the case in materials experiencing multiple cracking such as concrete, rocks, and brittle matrix composites. In this case, the previous descriptions are not valid, and it is necessary to take the crack orientation into account.

\section{2}

\section{Anisotropic description}

When studying the effect of a distribution of crack orientation, it is easier initially to limit the study to plane stress conditions. This is also a circumstance when it is an advantage to introduce the Gibbs specific enthalpy $\varphi$ following the Legendre transformation of Eq. (10). Figure 2 shows a sheet subjected 

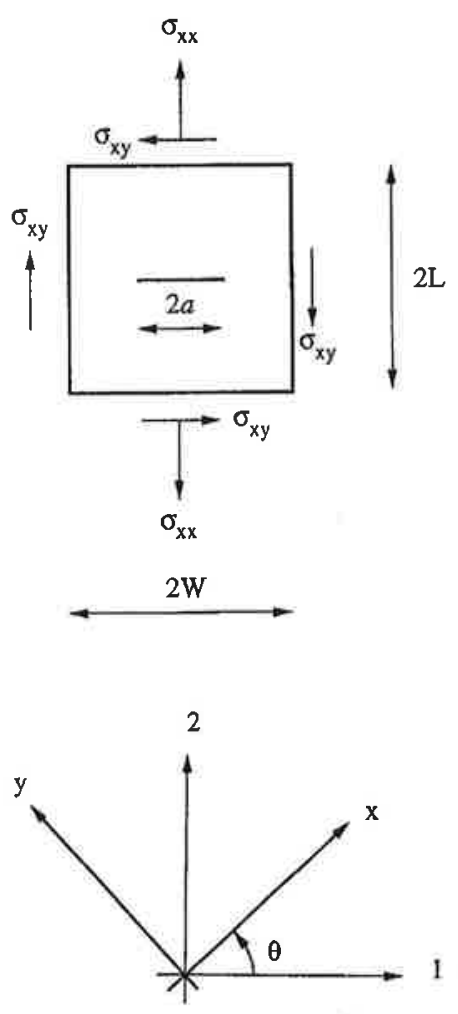

Fig. 3. The initial principal directions of orthotropy, or material directions, 11 and 2 often do not coincide with the loading directions $x$ and $y$. The angle $\theta$ measures their respective orientation

to a tensile stress $\sigma_{x x}$ and shear stress $\sigma_{x y}$ with a crack of length $2 a\left(\sigma_{x x}, \sigma_{y y}\right.$ and $\sigma_{x y}$ are the components of the stress tensor $\sigma$ of the material expressed in the $x-y$ frame, Fig. 3). The change of potential energy density due to the presence of a crack is given by [21]

$\Delta \varphi=\frac{\sigma_{x x}^{2}+\sigma_{x y}^{2}}{E} \omega_{c}(a)$

where $E$ is the Young's modulus of the virgin material, and $\omega_{c}(a)$ is the crack density assumed to be independent of the crack orientation $\mathbf{n}$. The corresponding compliance variation $\Delta \mathbf{S}$ at constant stress level is given by

$\Delta \mathbf{S}=\frac{\partial^{2} \Delta \varphi}{\partial \boldsymbol{\sigma} \partial \boldsymbol{\sigma}}$.

Since the energy density variation written in Eq. (34) is proportional to $\sigma_{x x}^{2}+\sigma_{x y}^{2}$ Eq. (35) shows that the compliance variation affects the Young's modulus along the $x$-direction as well as the shear modulus, so that the expression of the Gibbs specific enthalpy is

$2 \varphi=\frac{\sigma_{x x}^{2}}{E\left(1-D_{x}\right)}+\frac{2 v \sigma_{x x} \sigma_{y y}}{E}+\frac{\sigma_{y y}^{2}}{E}+\frac{\sigma_{x y}^{2}}{\tilde{G}\left(D_{x}\right)}$,

with

$\tilde{G}\left(D_{x}\right)=\frac{G}{1+\left(\frac{D_{x}}{1-D_{x}}\right) \frac{1}{2(1+v)}}$

and

$D_{x}=\frac{2 \omega_{c}(a)}{1+2 \omega_{c}(a)}$

where $E, v$ are the initial elastic properties of the matrix, $\widetilde{G}$ is the shear modulus which is a function of damage variable $D_{x^{*}}$ 
The associated force to the damage variable $D_{x}$ corresponds to the energy release rate density $Y_{x}$

$Y_{x}=\frac{\partial \varphi}{\partial D_{x}}=\frac{1}{2} \frac{\sigma_{x x}^{2}+\sigma_{x y}^{2}}{E\left(1-D_{x}\right)^{2}}$.

The corresponding Helmholtz free energy density is obtained as a Legendre-Fenchel transformation of Eq. (36)

$2 \psi=\frac{E\left(\left(1-D_{x}\right) \varepsilon_{x x}^{2}+2 v\left(1-D_{x}\right) \varepsilon_{x x} \varepsilon_{y y}+\varepsilon_{y y}^{2}\right)}{1-v^{2}\left(1-D_{x}\right)}+4 \tilde{G}\left(D_{x}\right) \varepsilon_{x y}^{2}$,

and the energy release rate density $Y_{x}$ is now defined as

$Y_{x}=-\frac{\partial \psi}{\partial D_{x}}$

and has the same significance and expression as that given in Eq. (39).

When interactions between cracks are accounted for, it is shown that within the framework of the Nonlocal Damage Theory (NDT) [30], the damage variable measured as loss of stiffness not only depends on the crack density $\omega_{c}$ but also on the interaction between cracks. For instance, in tension it is shown that the damage variable $D$ of cracked systems along one direction, measured as a loss of stiffness in the perpendicular direction, is given by

$D=\frac{H \omega_{c}}{1+H \omega_{c}}$,

with

$\omega_{c}=\frac{\pi a^{2}}{4 L W}$

where $2 L$ and $2 W$ denote the cell size as well as the distance between two horizontal and two vertical cracks. The interaction coefficient $H$ represents the interaction between the cracks in the considered array [31]. It is worth noting that Eq. (38) is similar to Eq. (43). However, depending on the micromechanical model, the relationship between the damage variable and the crack density is different.

The previous results can be generalized to materials with a distribution of crack size $a$ and orientations $\mathbf{n}$. This distribution is characterized by a probability density function $f(a, \mathbf{n})$. The average energy variation $\Delta \bar{\varphi}$ is then expressed as

$\Delta \bar{\varphi}=\frac{1}{2 \pi} \int_{\mathscr{G}}^{+\infty} \int_{0}^{+\infty} \Delta \varphi(a, \mathbf{n}) f(a, \mathbf{n}) \mathrm{d} a \mathrm{~d} l$,

where $\mathscr{C}$ is the circle of unit directions $\mathbf{n}$. If the interactions are neglected, the energy variation for a crack of size $a$ and orientation $\mathbf{n}$ is given by

$\Delta \varphi(a, \mathbf{n})=\omega_{c}(a) \frac{\mathbf{n} \cdot \boldsymbol{\sigma} \cdot \boldsymbol{\sigma} \cdot \mathbf{n}}{E}$.

The average compliance variation $\Delta \mathbf{S}$ at constant stress level is then given by

$\Delta \mathbf{S}=\frac{\partial^{2} \Delta \bar{\varphi}}{\partial \boldsymbol{\sigma} \partial \boldsymbol{\sigma}}=\frac{1}{2 \pi} \frac{1}{E} \int_{\mathscr{q}}\left\langle\omega_{c}(\mathbf{n})\right\rangle \mathbf{N}(\mathbf{n}) \mathrm{d} l$,

with

$\left\langle\omega_{c}(\mathbf{n})\right\rangle=\int_{0}^{+\infty} \omega_{c}(a) f(a, \mathbf{n}) \mathrm{d} a$,

$N_{\alpha \beta \gamma \delta}(\mathbf{n})=\frac{1}{2}\left(n_{\alpha} n_{\gamma} \delta_{\beta \delta}+n_{\alpha} n_{\delta} \delta_{\gamma \beta}+n_{\beta} n_{\gamma} \delta_{\alpha \delta}+n_{\beta} n_{\delta} \delta_{\alpha \gamma}\right) \quad \alpha, \beta, \gamma, \delta=1,2$ 
where $\delta$ is the Kronecker symbol. The average crack density $\left\langle\omega_{c}(\mathbf{n})\right\rangle$ along a normal $\mathbf{n}$ can be expanded through a modified Fourier representation [32]

$\left\langle\omega_{\epsilon}(\mathbf{n})\right\rangle=\omega_{0} 1+\omega_{\alpha \beta} f_{\alpha \beta}+\omega_{\alpha \beta \gamma \delta} f_{\alpha \beta \gamma \tilde{\delta}}+\cdots$,

where $1, f_{\alpha \beta}, f_{\alpha \beta \gamma \delta} \ldots$ are the basis functions, and $\omega_{0}, \omega_{\alpha \beta \beta}, \omega_{\alpha \beta \gamma \delta} \ldots$ are the Fourier coefficients. The basis functions $1, f_{\alpha \beta}$, and $f_{\alpha \beta \gamma \gamma}, \ldots$ are orthogonal to each other so that

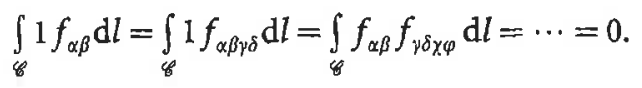

The tensors $f_{\alpha \beta}$ and $f_{\alpha \beta \gamma \delta}$ are completely symmetric with respect to their indices, traceless, and can be defined as follows:

$f_{\alpha \beta}(\mathbf{n})=n_{\alpha} n_{\beta}-\frac{1}{2} \delta_{\alpha \beta}$

$$
\begin{aligned}
f_{z \beta \gamma \delta}(\mathbf{n})= & n_{\alpha} n_{\beta} n_{\gamma} n_{\delta}-\frac{1}{6}\left(n_{\alpha} n_{\beta} \delta_{\gamma \delta}+n_{\gamma} n_{\delta} \delta_{\alpha \beta}+n_{\alpha} n_{\gamma} \delta_{\beta \delta}+n_{\alpha} n_{\delta} \delta_{\gamma \beta}+n_{\beta} n_{\gamma} \delta_{\alpha \delta}+n_{\beta} n_{\delta} \delta_{\alpha \gamma}\right) \\
& +\frac{1}{6 \times 4}\left(\delta_{\alpha \beta} \delta_{\gamma \delta}+\delta_{\alpha \gamma} \delta_{\beta \delta}+\delta_{\alpha \delta} \delta_{\beta \gamma}\right) .
\end{aligned}
$$

$\ldots$

The expansion coefficients $\omega_{\alpha \beta}, \omega_{\alpha \beta \gamma \delta}, \ldots$ are also completely symmetric and traceless with respect to their indices. They are expressed as the inner product of $\left\langle\omega_{c}(\mathbf{n})\right\rangle$ and the basis functions

$\omega_{0}=\frac{1}{2 \pi} \int_{\mathscr{C}}\left\langle\omega_{c}(\mathbf{n})\right\rangle \mathrm{d} l$

$\omega_{\alpha \beta}=\frac{1}{2 \pi} \frac{2 \times 4}{2} \int_{\mathscr{C}}\left\langle\omega_{c}(\mathbf{n})\right\rangle f_{\alpha \beta}(\mathbf{n}) \mathrm{d} l$,

$\omega_{\alpha \beta \gamma \delta \delta}=\frac{1}{2 \pi} \frac{2 \times 4 \times 6 \times 8}{2 \times 3 \times 4} \int_{\mathscr{C}}\left\langle\omega_{c}(\mathbf{n})\right\rangle f_{\alpha \beta \gamma \delta}(\mathbf{n}) d l$,

. .

These tensors are written for plane problems, the same formalism can be used in 3D problems as shown in $[32 ; 33]$.

According to Onat and Leckie [32-34], a fourth-order tensor, e.g. the stiffness tensor C, with the following symmetries

$C_{\alpha \beta \gamma \delta}=C_{\beta \alpha \gamma \delta}=C_{\alpha \beta \delta \gamma}=C_{\gamma \delta \alpha \beta}$,

can be written as a unique linear combination of the previous basis functions. The fourth-order tensor $N(n)$ can be expressed as a combination of the first two basis functions

$N_{\alpha \beta \gamma \delta}(\mathbf{n})=\frac{1}{2}\left(\delta_{\alpha \gamma} \delta_{\beta \delta}+\delta_{\alpha \delta} \delta_{\beta \gamma}\right)+\frac{1}{2}\left(f_{\alpha \gamma}(\mathbf{n}) \delta_{\beta \delta}+f_{\alpha \delta}(\mathbf{n}) \delta_{\gamma \beta}+f_{\beta \gamma}(\mathbf{n}) \delta_{\alpha \delta}+f_{\beta \delta}(\mathbf{n}) \delta_{\alpha \gamma}\right)$,

and the compliance variation depends upon the first two coefficients of the series expansion (47)

$\Delta \bar{S}_{\alpha \beta \gamma \delta}=\frac{1}{E}\left[\frac{1}{2} \omega_{0}\left(\delta_{\alpha \gamma} \delta_{\beta \delta}+\delta_{\alpha \delta} \delta_{\beta \gamma}\right)+\frac{1}{8}\left(\omega_{\alpha \gamma} \delta_{\beta \delta}+\omega_{\alpha \delta} \delta_{y \beta}+\omega_{\beta \gamma} \delta_{\alpha, \delta}+\omega_{\beta \delta} \delta_{\alpha \gamma}\right)\right]$.

Equation (53) shows that in the case of a random distribution of cracks, the first two coefficients of the series expansion (47) define the damage state variables. To get the expression of the Helmholtz free energy density, a Legendre-Fenchel transformation of the Gibbs specific enthalpy, has to be used.

These results are written for plane problems, and the same formalism can be used in 3D problems related to random distributions of penny-shaped cracks as shown in [32-34]. In that case, the first three coefficients of the series expansion of the average crack density $\left\langle\omega_{c}(\mathbf{n})\right\rangle$ along a normal n define the damage state variables. 
Inelastic properties of damaged materials

The inelastic strains which occur in CMCs are the result of slip at interfaces. The inelastic strains resulting from the slip and the elastic energy introduced into the broken part are the subjects of this section. The unit cell in Fig. 4 shows a crack in a unidirectionally reinforced CMC accompanied by interface slip occurring over a debond length $l_{F}$. The volume fraction of the broken part is $f$, and the elastic moduli of the broken part and the broken part are $E_{u}, G_{u}$ and $E_{b}, G_{b}$, respectively. There are two components of slip whose effects are discussed separately.

The first component of slip occurs when tensile stress is applied in the fiber direction. The faces of the crack are open by an amount $\Delta$ and cause slip over the debond length $l_{p}$, Fig. 4 . When the slip is introduced and locked in position, a self-equilibrating state of stress $\boldsymbol{\rho}^{\mu}$ is introduced into the composite and the total compatible strains consist of two components

$\varepsilon^{\mu}=\varepsilon^{\mu e}+\varepsilon^{\mu i n}$

where $\varepsilon^{\mu e}$ is the elastic strain tensor which satisfies the elastic stress/strain condition

$\varepsilon^{\mu e}=C^{\mu}: \rho^{\mu}$

and $\varepsilon^{\mu i n}$ are the inelastic strains. The macroscopic inelastic strains may be calculated using the relationship derived in [10]

$V \hat{\boldsymbol{\sigma}}: \alpha+\int_{A} \hat{\mathrm{T}}^{+} \cdot \mathbf{u} \mathrm{d} S=\int_{\Omega} \hat{\boldsymbol{\sigma}}^{\mu}:\left(\varepsilon^{\mu e}+\varepsilon^{\mu l n}\right) \mathrm{d} V$

where $\hat{\boldsymbol{\sigma}}^{\mu}$ is the elastic stress in the composite corresponding to a macro stress $\hat{\boldsymbol{\sigma}}$, and $\Omega$ an integration domain of volume $V$. The body $\Omega$ contains a plane of surface $A$ with outward normals $\mathbf{n}^{+}$and $\mathbf{n}^{-}$. The positive side of the plane moves an amount $\mathbf{u}$ with respect to the negative side. The body is treated as if it contained an internal surface $S$ that is subjected to tractions $\hat{\mathrm{T}}^{+}=-\hat{\boldsymbol{\sigma}} \cdot \mathrm{n}^{+}$along $S^{+}$ and $\hat{\mathrm{T}}^{-}=-\hat{\boldsymbol{\sigma}} \cdot \mathbf{n}^{-}$along $S^{-}$. When $\hat{\sigma}_{k l}=\hat{\sigma}_{11}=1$, the internal elastic stresses are

$\hat{\sigma}_{11}^{\mu u}=\frac{E_{u}}{E} \hat{\sigma}_{11}$

$\hat{\sigma}_{11}^{\mu b}=\frac{E_{b}}{E} \hat{\sigma}_{11}$

where $E=(1-f) E_{u}+f E_{b}$, then

$\alpha_{11}=\frac{\Delta}{L} \frac{f E_{b}}{E}$.

Similarly,

$\alpha_{22}=\alpha_{12}=0$

It might be noted that the same result applies irrespective of the crack angle.

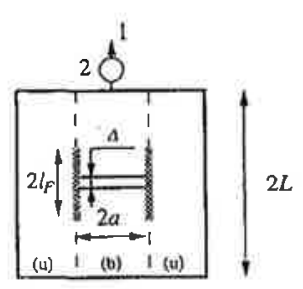

$2 W$

Fig. 4. Elementary cell in tension of size $2 L \times 2 W$ containing a crack of size $2 a$. A friction zone is characterized by the friction length $l_{F}$ 


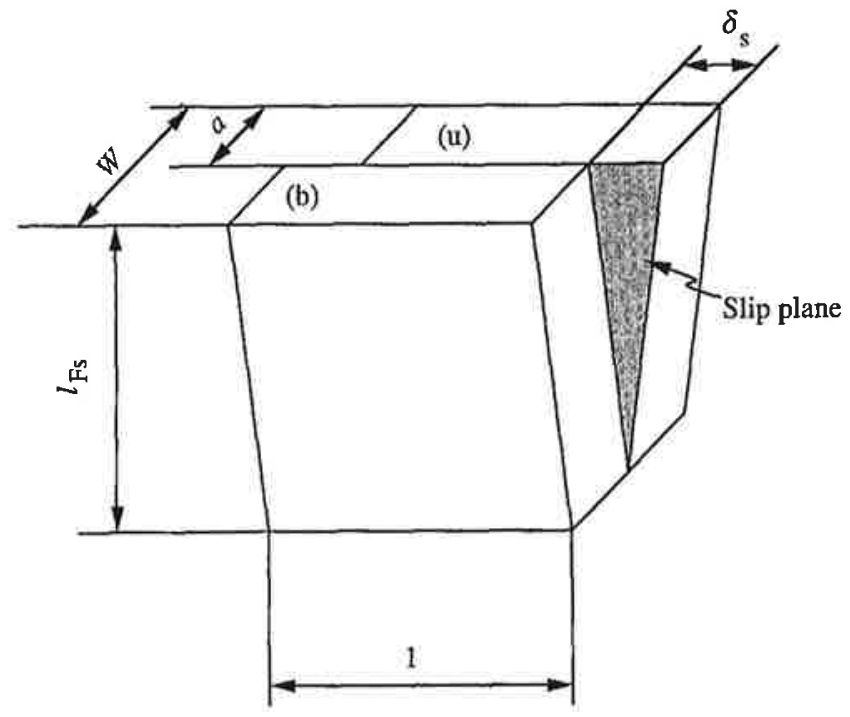

Fig. 5. Elementary cell in shear containing a crack of size $2 a$. A friction zone is characterized by the friction length $l_{\text {Ps }}$

The second type of slip which is the result of shear is shown in Fig. 5. Applying again the previous results, and using the shear field shown in Fig. 5, when $\hat{\sigma}_{k 1}=\hat{\sigma}_{t 2}=\hat{\sigma}_{21}=1$, for which the elastic stress distribution is

$\hat{\sigma}_{12}^{\mu u}=\frac{G_{u}}{G} \hat{\sigma}_{12}, \quad \hat{\sigma}_{12}^{\mu b}=\frac{G_{b}}{G} \hat{\sigma}_{12}$

gives the result

$\alpha_{12}=\frac{\delta_{s}}{L} \frac{f G_{b}}{G}$

$\alpha_{11}=\alpha_{22}=0$

where $G=(1-f) G_{u}+f G_{b}$. With the assumption on the shear stress at the slipping interface, it is possible to show that the elastic energy resulting from the slip $\Delta$ is

$\psi^{s}(\Delta)=\frac{2}{3} \frac{f E_{b}(1-f) E_{u}}{E}\left(\frac{\Delta}{l_{F}}\right)^{2} \frac{l_{F}}{L}$

and the corresponding value for the shear slip $\delta_{s}$ is

$\psi^{s}(\delta)=\frac{1}{2} \frac{f G_{b}(1-f) G_{u}}{G}\left(\frac{\delta}{l_{F s}}\right)^{2} \frac{l_{F s}}{L}$.

The results derived in Sect. 3 and 4 will be used in the following sections to derive a $2 \mathrm{D}$ constitutive law of CMCs under monotonic loading conditions.

5

CMCs with multidirectional fibers systems

This section is concerned with the behavior of ceramics reinforced by CMCs. It has been demonstrated by Evans and Marshall [35] that following matrix cracking, sliding occurs at the fiber/matrix interface which results in inelastic deformations. The presence of matrix cracks and inelastic deformations may impart to the material the ability to redistribute stresses. The results of experiments on notched panels on SiC/CAS composites [36] suggest the capacity of the material to redistribute stress is sufficiently high for this material to be notch-insensitive. The ability to redistribute stress is an important property since design studies indicate that working stresses are sufficiently high for matrix cracking to be unavoidable in regions of stress concentration. 
The micromechanics which describes interface bedonding and sliding has been established by Hutchinson and Jensen [37] and Evans et al. [38]. In contrast to the early phenomenological studies $[25 ; 39]$, the intention of the present study is to develop a continuum description of the damage processes which is mechanism-based, and which may be used to describe the behavior of CMCs under the conditions of multiaxial stresses occurring in practice. Since crack spacing at saturation is small in CMCs [12], CDM is an appropriate means of describing damage. Changes in elastic moduli, measured on a macroscopic level, provide a simpler and more robust means of measuring damage than does microscopic measurement of crack density. The latter requires the average of many readings before reliable values are established [40].

By combining CDM with the micromechanical studies referred to previously, constitutive equations are developed which lend themselves to the finite element procedures commonly used in practice $[41 ; 42]$. Following established procedures, the properties of each layer are first derived, and those of the composite are calculated by ensuring compatibility conditions.

The components of each layer consist of the matrix, the fiber and the interface, with $f$ being the fiber volume fraction. The fiber direction defines the $1-2$ axes. The axes $x-y$ correspond to the principal axes of the strains in the ceramic matrix. The definition of the axes used at the constituent, layer and composite levels are shown in Fig. 3. Following Sect. 3, the loss of stiffness due to matrix cracking and fiber breakage is first established, and this is followed by the influence of the slip at the interface studies in Sect. 4.

\section{1}

\section{Elastic energies of the composite associated with matrix cracking and fiber breakage}

\subsection{1}

\section{Constituent level: matrix and fiber}

The initial behavior of the matrix is assumed to be isotropic. The presence of cracks leads to an anisotropic behavior. The assumption is made that cracking occurs in the $x$-direction (e.g. the maximal principal strain direction) in the matrix, and that this direction is constant during a monotonic load history. It is worth noting that this assumption excludes nonproportional loading conditions as well as equibiaxial straining. Under this hypothesis, only one damage variable for matrix cracking is needed, and is denoted by $D_{m x}$. The crack distribution is different from zero only along one direction. The results of Sect. 3 show that the Young's modulus along each direction as well as the shear modulus are altered $[21 ; 43]$. The expression of the elastic energy density of the matrix is (see Eq. (40))

$\psi_{m}=\frac{1}{2} \frac{E_{m}\left(\left(1-D_{m x}\right) \varepsilon_{m x x}^{2}+2 v_{m}\left(1-D_{m x}\right) \varepsilon_{m x x} \varepsilon_{m y y}+\varepsilon_{m y y}^{2}\right)}{1-v_{m}^{2}\left(1-D_{m x}\right)}+2 \tilde{G}_{m}\left(D_{m x}\right) \varepsilon_{m x y}^{2}$,

with

$$
\tilde{G}_{m}\left(D_{m x}\right)=\frac{G_{m}}{1+\frac{D_{m x}}{1-D_{m x}} \frac{1}{2\left(1+v_{m}\right)}}
$$

where $E_{m}, v_{m}$ are the initial elastic properties of the matrix, $\tilde{G}_{m}$ is the shear modulus which is a function of damage variables $D_{m x x}$, and $\varepsilon_{m \times x}, \varepsilon_{m y y}, \varepsilon_{m x y}$ are the components of the strain tensor $\varepsilon_{m}$ of the matrix expressed in the $x-y$ frame.

It is assumed that the fibers are aligned along the 1-direction and that fiber breakage is perpendicular to the fiber direction. Therefore the elastic energy is given by

$\psi_{f}=\frac{1}{2}\left[E_{f}\left(1-D_{f 1}\right) \varepsilon_{f 11}^{2}+E_{f} \varepsilon_{f 22}^{2}\right]+2 G_{f}\left(D_{f 1}\right) \varepsilon_{f 12}^{2}$

where $E_{f}$ is the Young's modulus of the uncracked fiber, $G_{f}\left(D_{f}\right)$ is the shear modulus of the cracked fiber embedded in the matrix, $\varepsilon_{f 11}, \varepsilon_{f 22}$ and $\varepsilon_{f 12}$ the components of the strain tensor $\varepsilon_{f}$ of the fiber expressed in the 1-2 frame.

The expression of the stresses in the matrix $\sigma_{m}$ and in the fibers $\sigma_{f}$ are obtained by partial differentiation of the elastic energy density with respect to the strain tensors $\varepsilon_{m}$ and $\varepsilon_{f}$, respectively,

$$
\begin{aligned}
& \boldsymbol{\sigma}_{m}=\frac{\partial \psi_{m}}{\partial \boldsymbol{\varepsilon}_{m}}, \\
& \boldsymbol{\sigma}_{f}=\frac{\partial \psi_{f}}{\partial \boldsymbol{\varepsilon}_{f}},
\end{aligned}
$$


and the associated forces are defined as

$Y_{m x}=-\frac{\partial \psi_{m}}{\partial D_{m x}}$,

$Y_{f 1}=-\frac{\partial \psi_{f}}{\partial D_{f 1}}$.

These generalized forces are the energy release rate densities associated with matrix cracking and fiber breakage, respectively.

\section{1 .2}

Layered composite

When the composite consists of layers of unidirectional fibers with different orientations, the laminate properties are determined by applying laminate theory to the properties of individual layers.

\subsubsection{1}

\section{Layer level}

A layer consists of fibers aligned along one orientation embedded in a matrix. To determine the behavior of this layer, micro-interface compatibility conditions are written in terms of the strains $\varepsilon^{L}$ and stresses $\sigma^{L}$ on the layer level. Those conditions are the compatibility and the equilibrium between a fiber and the surrounding matrix, which takes place in that system. Therefore, it is more convenient to write the conditions in the 1-2 material frame as follows:

$$
\begin{aligned}
& \varepsilon_{m 11}=\varepsilon_{f 11}=\varepsilon_{11}^{L}, \\
& f_{m} \sigma_{m 11}+f_{f} \sigma_{f 11}=\sigma_{11}^{L}, \\
& f_{m} \varepsilon_{m 22}+f_{f} \varepsilon_{f 22}=\varepsilon_{22}^{L}, \\
& \sigma_{m 22}=\sigma_{f 22}=\sigma_{22}^{L}, \\
& f_{m} \varepsilon_{m 12}+f_{f} \varepsilon_{f 12}=\varepsilon_{12}^{L}, \\
& \sigma_{m 12}=\sigma_{f 12}=\sigma_{12}^{L},
\end{aligned}
$$

where $f_{f}$ denotes the fiber volume fraction, and $f_{m}$ denotes the matrix volume fraction. When the principal strain directions do not coincide with the material frame, Eqs. (73-78) have to be rewritten in the 1-2 frame. The application of these equations then defines the elastic properties of the layer,

$\sigma^{L}=\mathrm{E}^{L}\left(D_{m x}, D_{f 1}\right): \varepsilon^{L}$

where $\mathbf{E}^{L}\left(D_{m x}, D_{f 1}\right)$ is the fourth order elastic tensor of the layer level, and is a function of all damage variables at the constituent level. From Eq. (79), the elastic energy density associated with matrix cracking and fiber breakage on the layer level can be written as

$\psi^{L}=\frac{1}{2} \varepsilon^{L}: \mathbf{E}^{L}\left(D_{m x}, D_{f 1}\right): \varepsilon^{L}$

\section{1 .2 .2}

\section{Composite level}

For simplicity, the case of two layers at 0 and 90 degrees is considered. The micromechanical quantities associated with the 0 degree layer are superscripted by ${ }^{00}$, and those at 90 degrees by ${ }^{90}$. The elastic behavior of the composite system is determined by applying the classical laminate theory. The compatibility condition and the overall stresses $\sigma$ are

$$
\begin{aligned}
& \varepsilon=\varepsilon^{00}=\varepsilon^{90}, \\
& \sigma=f^{00} \sigma^{00}+f^{90} \sigma^{90},
\end{aligned}
$$


where $f^{00}$ and $f^{90}$ denote, respectively, the volume fraction of the first and the second layer, $\boldsymbol{\sigma}^{00}$ and $\sigma^{90}$ denote, respectively, the stress tensor of the first and the second layer. By solving Eqs. (73-78) and using Eqs. (81-82), the overall behavior of the composite is defined as

$\boldsymbol{\sigma}=\mathbf{E}\left(D_{m x}^{00}, D_{f 1}^{00}, D_{m x}^{90}, D_{f 1}^{90}\right): \varepsilon$,

with

$\mathrm{E}\left(D_{m x}^{00}, D_{f 1}^{00}, D_{m x}^{90}, D_{f 1}^{90}\right)=f^{00} \mathrm{E}^{00}\left(D_{m x}^{00}, D_{f 1}^{00}\right)+f^{90} \mathbf{E}^{90}\left(D_{m x}^{90}, D_{f 1}^{90}\right)$,

where $\mathbf{E}\left(D_{m x}^{\infty 0}, D_{f 1}^{\infty 0}, D_{m x}^{90}, D_{f i}^{90}\right)$ is the fourth order elastic tensor on the composite level, which is a function of all damage variables $D_{m x}^{o 0}, D_{f 1}^{00}, D_{m x}^{90}, D_{f 1}^{90}$ on the constituent level for the two layers. From Eqs. (81-82), the elastic energy associated with matrix cracking and fiber breakage on the composite level can be written as

$\psi^{D}=f^{00} \psi^{00}+f^{90} \psi^{90}$

\section{2}

\section{State potential associated with debonding and fiber Pull out}

Inelasticity is essentially due to sliding at the interface between the fiber and the matrix. Sliding is involved in debonding as well as fiber pullout. From a micromechanical point of view, this sliding can take place as soon as a crack is bridged by fibers. In a CDM formulation, only the equivalent homogenous sliding and the associated forces are considered. Therefore, the cell model used to describe cracking and sliding is that shown in Figs. 4, 5. The analysis that has been done in Sect. 4 to give the expression of the elastic energy due to sliding of a layered composite along the 1-2 directions can be extended as follows:

$\psi^{s}=\frac{1}{2} E\left(\frac{\alpha_{11}^{2}}{d_{11}}+\frac{\alpha_{22}^{2}}{d_{22}}\right)+\frac{1}{2} G\left(\frac{\alpha_{12}^{2}}{d_{12}}\right)$

with

$E=\frac{4}{3} \frac{f^{00} E^{00} f^{90} E^{90}}{f^{00} E^{00}+f^{90} E^{90}}, \quad G=\frac{f^{00} G^{00} f^{90} G^{90}}{f^{00} G^{00}+f^{90} G^{90}}$,

where $E^{00}$ is the Young's modulus of the 0-degree layer in the fiber direction $00, E^{90}$ is the Young's modulus of the 90 degree layer in the fiber direction $90, G^{00}$ is the shear modulus of the 0-degree layer, $G^{90}$ is the shear modulus of the 90-degree layer, $d_{11}, d_{22}$ and $d_{12}$ are damage quantities related to sliding, and $\alpha_{11}, \alpha_{22}$ and $\alpha_{12}$ are the inelastic strains.

\subsection{1}

\section{State laws}

The following development deals with ceramic matrix layered composites. The total elastic energy density of the composite is the sum of the elastic energy density of the damaged composite $\psi^{D}$ and the elastic energy density due to sliding $\psi^{s}$

$\psi=\frac{1}{2}(\varepsilon-\alpha): \mathbf{E}\left(D_{m x}^{00}, D_{m x}^{90}, D_{f 1}^{00}, D_{f 1}^{90}\right):(\varepsilon-\alpha)+\psi^{s}$,

where $D_{m}^{00}, D_{m}^{90}, D_{f,}^{00}$, and $D_{f 2}^{90}$ are the damage variables modeling matrix cracking and fiber breakage in the 0 and 90 degree plies. The forces associated with total strains are

$\boldsymbol{\sigma}=\frac{\partial \psi}{\partial \boldsymbol{\varepsilon}}=\mathbf{E}\left(D_{m x}^{00}, D_{m x}^{90}, D_{f 1}^{00}, D_{f 1}^{90}\right):(\varepsilon-\alpha)$

and correspond to the macroscopic stresses. The associated forces to the damage variables modeling matrix cracking and fiber breakage are

$Y=-\frac{\partial \psi}{\partial D}$, 
where $D=\left\{D_{m x}^{00} ; D_{m x}^{90} ; D_{f 1}^{00} ; D_{1}^{90}\right\}$, and $Y=\left\{Y_{m x}^{00}, Y_{m x}^{90}, Y_{f 1}^{00}, Y_{f 1}^{90}\right\}$ represent the energy release rate densities due to matrix cracking and fiber breakage. The associated forces to the damage variables modeling sliding are

$y=-\frac{\partial \psi}{\partial \mathbf{d}}$

where $\mathrm{d}=\left\{d_{11} ; d_{22} ; d_{12}\right\}$ and $\mathrm{y}$ correspond to the energy release rate densities due to sliding. The associated forces to the anelastic strains are

$\mathrm{X}=\frac{\partial \psi}{\partial \alpha}$

and represent the back stresses in the sliding zone.

\section{2 .2}

\section{Evolution laws}

\subsubsection{1}

\section{$1 D$ evolution law of a CMC}

The aim of this section is to derive the evolution laws from a 1D analysis. Postmortem analysis of broken specimens indicates the presence of arrays of microcracks in the matrix accompanied by debonding or friction at the fiber/matrix interface. Hutchinson and Jensen [37] and Evans et al. [38] have analyzed the behavior of a unidirectional CMC in tension by considering the unit cell shown in Fig. 4 , when matrix cracking of spacing $2 L$ is accompanied by sliding at the interface over a debond length $2 l_{f}$. The micromechanics can predict the $1 \mathrm{D}$ macroscopic stress-strain response shown in Fig. 6. The microcracks are usually aligned with the principal stress or strain directions. By studying a cracked panel with crack of length $2 a$ in a cell of area $4 L W$ (Fig. 2), the reduction in stiffness may be estimated. If the initial behavior of the elementary cell is isotropic, and the Young's modulus is $E$, as shown earlier, the stiffness loss depends on the crack density defined as $\pi a^{2} / 4 L W$. By assuming plane stress conditions, and that the crack density is so small that crack interaction can be neglected, a first approximation for the reduced elastic modulus $\tilde{E}$ can be written as (see Sect. 2)

$$
\frac{\tilde{E}}{E}=\frac{1}{1+2 \frac{\pi a^{2}}{4 L W}}=1-D \text {. }
$$

In the case of constituents with different elastic properties, $D$ depends upon the elastic properties of the two constituents, as well as the ratios $a / W$ and $a / L$. The stress/strain relationship becomes

$$
\sigma=\frac{\bar{\sigma}}{1-D}=E \bar{\varepsilon},
$$

where $\sigma$ is the stress on a microscopic level, $\bar{\sigma}$ and $\bar{\varepsilon}$ are the macroscopic stress and strain, respectively. The model illustrated in Fig. 4 is now analyzed and uses an analytic approach which follows the thermodynamic developments summarized in Sect. 2 . This is done by calculating the internal elastic density $\psi^{s}$ in the unit cell [44] caused by debonding and sliding at the interface. The first

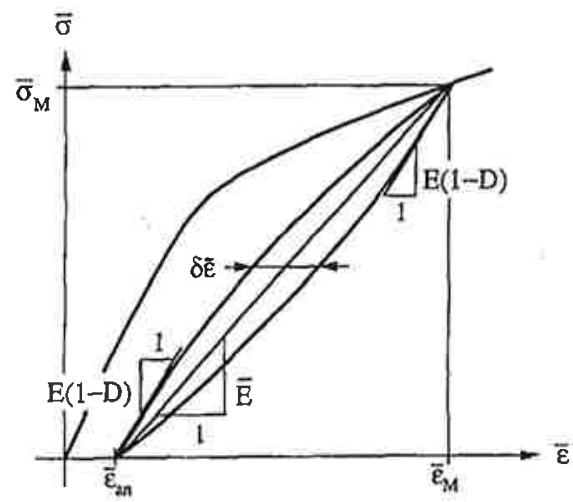

Fig. 6. Stress, $\vec{\sigma}$, versus strain, $\bar{\varepsilon}$, during a loading-unloadingreloading sequence 
step consists in moving the unbroken part (u) with respect to the broken part (b) with no external load by an amount $\Delta$ over a length $l_{F}$ (Fig. 7). The displacement $\Delta$ gives rise to a self-balanced linear stress field along a length $l_{F}$ in parts (b) and (u). By integration over $l_{F}$, and then by averaging over the total length $L$, the elastic energy density associated with this process is given by

$\psi^{s}=\frac{2}{3} \frac{f E_{b}(1-f) E_{u}}{E}\left(\frac{\Delta}{l_{F}}\right)^{2} \frac{l_{F}}{L}$

As shown in Eq. (59), the crack opening displacement $\Delta$ induces an irreversible strain $\alpha$ expressed as

$\alpha=\frac{f E_{b}}{E} \frac{\Delta}{L}$.

The second step consists of an elastic loading of the damaged system so that the elastic energy density is given by

$\psi^{e}=\frac{1}{2} E(1-D)(\bar{\varepsilon}-\alpha)^{2}$.

The total elastic energy density is the sum of the two elements of the energy densities so that

$\psi=\frac{1}{2} E(1-D)(\bar{\varepsilon}-\alpha)^{2}+\frac{2}{3} \frac{f E_{b}(1-f) E_{u}}{E}\left(\frac{\Delta}{l_{F}}\right)^{2} \frac{l_{F}}{L}$.

For convenience, the energy density can be expressed in a more compact form by using state variables which are the total strain $\bar{\varepsilon}$, the damage variable $D$ modeling the loss of stiffness due to the cracking mechanism, the damage variable $d=f E_{b} l_{F} /(1-f) E_{u} L$ which defines the size of the slip zone related to the crack spacing, and the crack opening strain $\alpha$. The elastic energy density in terms of the new internal variables is

$\psi=\frac{1}{2} E(1-D)(\bar{\varepsilon}-\alpha)^{2}+\frac{2}{3} E\left(\frac{\alpha^{2}}{d}\right)$.

The forces associated with the state variables $(\bar{\varepsilon}, D, d, \alpha)$ are respectively given by

$\bar{\sigma}=\frac{\partial \psi}{\partial \bar{\varepsilon}}=E(1-D)(\bar{\varepsilon}-\alpha)$,

$Y=-\frac{\partial \psi}{\partial D}=\frac{E}{2}(\bar{\varepsilon}-\alpha)^{2}$,

$y=-\frac{\partial \psi}{\partial d}=\frac{2 E}{3}\left(\frac{\alpha}{d}\right)^{2}$,

$X=\frac{\partial \psi}{\partial \alpha}=-\bar{\sigma}+\frac{4 E}{3} \frac{\alpha}{d}$

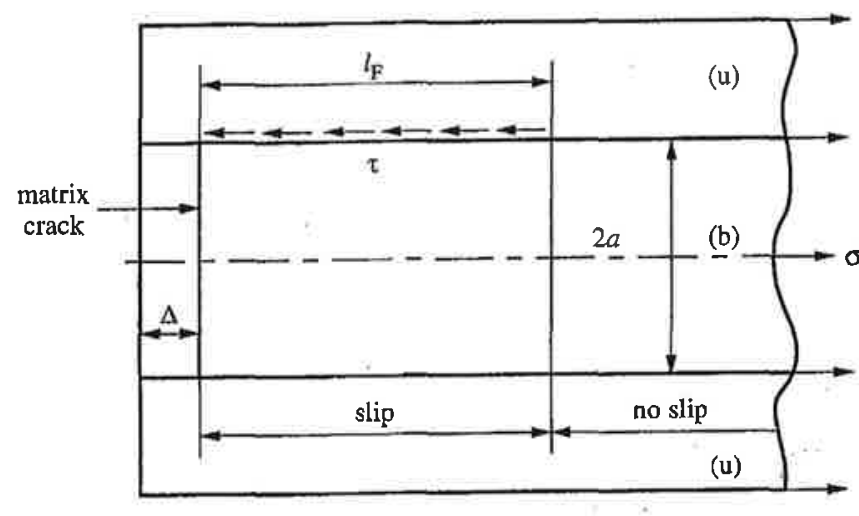

Fig. 7. Motion of the unbroken part (u) with respect to the broken part (b) with no external load by an amount $\Delta$ over a length $l_{p}$ 
where $\vec{\sigma}$ is the applied stress, $Y$ is the energy released rate available to form matrix cracks, $y$ the energy release rate available for interface debonding and $X$ is the back-stress induced by the slipping mechanism.

In the present approach the growth laws of the state variables $(D, d, \alpha)$ are established from macroscopic quantities measured in the course of unloading and reloading experiments. To this end, use is made of the solution [44] of the response of the unit cell (Fig. 4) when subjected to an unloading/reloading cycle during which the magnitude of the shear stress remains constant.

The expressions obtained from the analysis for the residual stress $\rho_{b}$ and the internal variables $D, d, \alpha$ in terms of the macroscopic quantities shown in Fig. 6 are given respectively by

$\frac{-\rho_{b}}{E}=\left(\sqrt{\frac{\bar{\varepsilon}_{\text {in }}+2 \delta \bar{\varepsilon}}{4 \delta \bar{\varepsilon}}}-1\right)\left(\bar{\varepsilon}_{M}-\bar{\varepsilon}_{\text {in }}-2 \delta \bar{\varepsilon}\right)$,

$D=\frac{\bar{\varepsilon}_{M} \bar{D}-\bar{\varepsilon}_{i n} \bar{D}-2 \delta \bar{\varepsilon}}{\bar{\varepsilon}_{M}-\bar{\varepsilon}_{i n}-2 \delta \bar{\varepsilon}}$,

$\frac{d}{4}=\frac{\sqrt{\left(\bar{\varepsilon}_{i n}+2 \delta \bar{\varepsilon}\right) \delta \bar{\varepsilon}}}{\bar{\varepsilon}_{M}-\bar{\varepsilon}_{\text {in }}-2 \delta \bar{\varepsilon}}$,

$\alpha=\frac{d}{2} \frac{\bar{\sigma}_{M}-\rho_{b}(1-D)}{E(1-D)}$

where $-\rho_{b} E / E_{b}$ is the thermal mismatch residual stress in the broken layer, $\bar{\varepsilon}_{i n}$ is the inelastic strain upon complete unloading, $\delta \bar{\varepsilon}$ is the maximum hysteresis loop width, $E$ is the Young's modulus of the composite, $\bar{\varepsilon}_{M}\left(\bar{\sigma}_{M}\right)$ is the maximum applied strain (stress), $D$ and $\bar{D}$ are, respectively, the microscopic and macroscopic damage associated with matrix cracking, $d$ is the damage related to interface debonding and $\alpha$ is the inelastic strain at the current state point. Eq. (106) is only valid for monotonic loading conditions.

By performing a series of unloading/reloading cycles, the internal variables can be determined from experiment using Eqs. (103-106). The residual stress $\rho_{b}$ is calculated from Eq. (103), and it is a test of the effectiveness of the model that the same value of the residual stress is obtained for each loading sequence. The values of $D$ and $d$ are given by applying Eqs. (104) and (105), respectively. The information is now available to complete the calculation for Eq. (106). The corresponding associated forces are given by Eqs. $(99-102)$. The relationship between the internal variables and the associated quantities can then be investigated. It is this method which is proposed to model the behaviox of the CMC laminate [44].

\subsubsection{2}

\section{Indentification of a 2D evolution law of a CMC}

The identification procedure is performed on a $[0 / 90]_{s}$ laminate architecture of CMCs. The first step is to define all the internal state variables needed to model the material behavior. The three strain variables, $\left\{\varepsilon_{11}, \varepsilon_{22}, \varepsilon_{12}\right\}$, are given either from experiment or as input from a FE calculation. The four damage variables, i.e. $\left\{D_{m x}^{00}, D_{m x}^{90}, D_{f 1}^{00}, D_{f 1}^{90}\right\}$ are used to define the change in the elastic properties, where $D_{m}$ is matrix-cracking damage, and fiber breakage damage is $D_{f}$. Assuming the damage evolution laws, $D_{m}\left(Y_{m}\right)$ and $D_{f}\left(Y_{f}\right)$, are functions of the associated forces $Y_{m}$ and $Y_{f}$, respectively, then only two evolution laws, one for each mechanism, are sufficient to compute the four components of damage. The three damage variables, $\left\{d_{11} ; d_{22} ; d_{12}\right\}$, define the sliding distances, with $d_{11}$ or $d_{22}$ being associated with sliding in the fiber directions, and $d_{12}$ associated with shear sliding. Consequently, only two evolution laws are needed, $d_{11}(\mathrm{y})$ or $d_{22}(\mathrm{y})$, and $d_{12}(\mathrm{y})$.

For the same reasons, two evolution laws for the inelastic strains, $\alpha_{11}(X)$ or $\alpha_{22}(X)$, and $\alpha_{12}(X)$ give the evolution of the three inelastic strains, $\left\{\alpha_{11}, \alpha_{22}, \alpha_{12}\right\}$. In conclusion, the model has 13 state variables, three of which are strain inputs and the remaining ten micromechanical state variables are derived from six evolution laws.

The second step is to define the relevant tests required to identify the growth of the six state variables. This is achieved from unloading tests performed at regular intervals and measuring the macroscopic inelastic strain upon complete unloading, $\vec{\varepsilon}_{i n}$, the macroscopic damage, of the composite, $\bar{D}$, and the maximum hysteresis loop width, $\delta \bar{\varepsilon}$ (Fig. 6). Using Eqs. (88-91), the internal states can be calculated. 
When tension is applied at 45 degrees on a [0/90], layered composite, only matrix cracking occurs. The macroscopic damage, $\widetilde{D}^{45}$, is related to the microscopic damage variables taking place in the matrix alone, $\left\{D_{m x}^{00} ; D_{m x}^{90}\right\}$. Moreover, the loading condition is such that the two damage variables, $D_{m x}^{\infty 0}$ and $D_{m x}^{90}$, have the same value. Therefore, the evolution law, $D_{m}\left(Y_{m}\right)$, is directly given by the evolution of the macroscopic damage of the composite $\bar{D}^{45}$.

The evolution law of the damage variable associated with fiber breakage, $D_{f}\left(Y_{f}\right)$, is found from measurement of the macroscopic damage, $\bar{D}^{00}$, in a tension test at 0 degrees on the $[0 / 90]_{\text {s }}$ composite. This has been compensated by the contribution of matrix cracking which may be calculated from the results of the first test. Similarly, the evolution laws of the state variables related to sliding, $\alpha_{11}(\mathrm{X})$ or $\alpha_{22}(\mathbf{X})$, and $d_{11}(\mathrm{y})$ or $d_{22}(\mathrm{y})$, are known from the evolution of the macroscopic inelastic strain upon complete unloading, $\bar{\varepsilon}_{i n}^{00}$, the macroscopic damage of the composite, $\bar{D}^{00}$, and the maximum hysteresis lopp width, $\delta \bar{\varepsilon}^{00}$, using micromechanics relations (103-106)

$$
\begin{aligned}
& \frac{-\rho_{b}^{00}}{E^{00}}=\left(\sqrt{\frac{\bar{\varepsilon}_{i n}^{00}+2 \delta \bar{\varepsilon}^{00}}{4 \delta \bar{\varepsilon}^{00}}}-1\right)\left(\bar{\varepsilon}_{m}^{00}-\bar{\varepsilon}_{i n}^{00}-2 \delta \bar{\varepsilon}^{00}\right), \\
& D^{00}=\frac{\vec{\varepsilon}_{M 1}^{00} \bar{D}^{00}-\bar{\varepsilon}_{i n}^{00} \bar{D}^{00}-2 \delta \bar{\varepsilon}^{00}}{\bar{\varepsilon}_{M}^{00}-\bar{\varepsilon}_{i n}^{00}-2 \delta \bar{\varepsilon}^{-00}} \\
& \frac{d_{11}}{4}=\frac{\sqrt{\left(\bar{\varepsilon}_{i n}^{00}+2 \delta \bar{\varepsilon}^{-00}\right) \delta \bar{\varepsilon}^{00}}}{\bar{\varepsilon}_{M}^{00}-\bar{\varepsilon}_{i n}^{00}-2 \delta \bar{\varepsilon}^{-00}} \\
& \alpha_{11}=\frac{d_{11}}{2} \frac{\bar{\sigma}_{M}^{00}-\rho_{b}^{00}\left(1-D^{00}\right)}{E^{00}\left(1-D^{00}\right)}
\end{aligned}
$$

where $\rho_{b}^{00}$ is proportional to the residual stress in the broken part of the 0 degree layer. Finally, returning to the results of the tension test at 45 degrees on a [0/90], layered composite, the evolution laws of the state variables related to sliding, $\alpha_{12}(X)$ and $d_{12}(y)$ are given by the following relationships similar to those calculated by the micromechanical analysis:

$$
\begin{aligned}
& \frac{d_{12}}{4}=\frac{\bar{\varepsilon}_{i n}^{45}}{\bar{\varepsilon}_{M}^{45}-2 \bar{\varepsilon}_{i n}^{45}}, \\
& \alpha_{12}=\frac{d_{12}}{2}-\frac{\bar{\varepsilon}_{M}^{45}}{1+\frac{d_{12}}{2}} .
\end{aligned}
$$

In this analysis, it can be noticed that the residual stress $-\rho_{b}^{45}$ in the tensile direction is equal to zero, and, therefore, $\bar{\varepsilon}_{i n}^{45}=2 \delta \bar{\varepsilon}^{45}$, which leads to the above results.

In summary, only two tests, on the same architecture, enable us to extract all six evolution laws that define the behavior of the material as shown in [45] on experiments performed on $\mathrm{SiC} / \mathrm{SiC}$ specimens [46].

\section{6}

\section{Conclusions}

CDM has grown out of the need to predict the behavior of engineering components composed of materials whose properties degrade because of damage. The traditional approach, which is now well established as a design procedure, has been formulated with only minimal attention to the internal mechanisms occuring in the material. In the above presentation, the formulation of classical CDM has been extended so that the description of the physical mechanisms which cause damage can be included. This approach has the advantage that the studies of material scientists are integrated into a formulation retaining the proven advantages of CDM. The illustrative examples of the application of the procedure is concerned with the properties of $\mathrm{CMC}$ which can suffer matrix cracking and fiber/matrix sliding resulting in a loss of stiffness, the appearance of inelastic deformations, as well as hysteresis loops. 


\section{References}

1. Kachanov, L. M.: Time of the rupture process under creep conditions. Izv. Akad. Nauk. S.R.S. Odt. Tekh. Nauk. 8 (1958) 26-31

2. Krajcinovic, D.: Damage mechanics. Mech. Mat. 8 (1989) 117-197

3. Lemaitre, J.: A course on damage mechanics. Berlin: Springer 1992

4. Rabotnov, Y. N.: On the equation of state for creep. In Koiter, W. T. (ed) Prager Anniversary Vol. (1963) pp. 307-315, McMillan

5. Lemaitre, J.: How to use damage mechanics. Nucl. Eng. Design 80 (1984) 233-245

6. Amar, G.; Dufailly, J.: Identification and validation of viscoplastic and damage constitutive equations. Eur. J. Mech. A/Solids 12 (1993) 197-218

7. Lemaitre, J.; Dufailly, J.: Damage measurements. Eng. Fract. Mech. 28 (1987) 643-661

8. Mazars, J.: Application de la mécanique de l'endommagement au comportement non linéaire et à la rupture du béton de structure. Thèse d'état, 1984

9. Ashby, M. F.; Dyson, B. F.: Creep damage mechanics and micromechanisms. Natl. Phys. Lab. UK 77, 1984

10. Cocks, A. C. F.; Leckie, F. A.: Creep constitutive equations for damaged materials. Adv. Appl. Mech. 25 (1987) 239-294

11. Hall, F. R.; Hayhurst, D. R.: Continuum damage mechanics modeling of high temperature deformation and failure in a pipe weldment. Proc. R. Soc. London/A Vol. 433 (1991) 383-403

12. Evans, A. G.: Perspectives on the development of high-thoughness ceramics. J. Am. Ceram. Soc. 73 (1990) 187-206

13. Rice, J. R.: Inelastic constitutive relations for solids: An internal variable theory and its application to metal plasticity. J. Mech. Phys. Solids 19 (1971) $433-455$

14. Bataille, J.; Kestin, J.: Irreversible processes and physical interpretations of rational thermodynamics. J. Non Equil. Thermodynamics 4 (1979) 229-258

15. Germain, P.; Nguyen, Q. S.; Suquet, P.: Continuum thermodynamics. Trans. ASME/J. Appl. Mech. 50 (1983) $1010-1020$

16. Volterra, V.: Sur l'équilibre des corps élastiques multiplement connexes. Annales Scientifiques Ecole Normale Supérieure 24 (1907) 401-518

17. Love, A. E. H.: The mathematical theory of elasticity. Cambridge: Cambridge University Press 1927

18. Eshelby, J, D.: The determination of the elastic field of an ellipsoidal inclusion and related problems. Proc. R. Soc. London/A 241 (1957) 376-396

19. Lemaitre, J.; Marquis, D.: Modeling complex behavior of metals by the 'State-Kinetic Coupling Theory'. J. Eng. Mat. Tech. 114 (1992) 250-254

20. Budiansky, B.: Thermal and thermoelastic properties of isotropic composites. J. Comp. Mater. 4 (1970) 286

21. Budiansky, B.; O’Connell, R. J.: Elastic moduli of a cracked system. Int. J. Solids Struct. 12 (1976) 81 -97

22. Lemaitre, J.; Chaboche, J.-L.: Aspect phénoménologique de la rupture par endommagement. J. Mec. Appl. 2 (1978) $317-365$

23. Lemaitre, J.: Sur la détermination des lois de comportement des matériaux élasto-viscoplastiques. Thèse de doctorat d'état, 1971

24. Chaboche, J.-L.: Description thermodynamique et phénoménologique de la viscoplasticité cyclique avec endommagement. Thèse d'état, 1978

25. Ladevèze, P.: Sur une théorie de l'endommagement anisotrope. LMT Cachan Report N. 34, 1983

26. Hill, R.: A self-consistent mechanics of composite materials. J. Mech. Phys. Solids 13 (1965) 213-222

27. Stolz, C.; Zaoui, A.: Combined variational approach and morphological analysis to the behavior of inhomogeneous elastic media, C. R. Acad. Sci. Paris 312 Série II (1991) 143-150

28. Christensen, R. M.; Lo, K. H.: Solutions for effective shear properties in three phase sphere and cylinder models. J. Mech. Phys. Solids 27 (1979) 315-330

29. Christensen, R. M.; Lo, K. H.: Solutions for effective shear properties in three phase sphere and cylinder models (erratum). J. Mech. Phys. Solids 34 (1986) 639

30. Pijaudier-Cabot, G.; Bazant, Z. P.: Nonlocal damage theory. J. Eng. Mech. 113 (1987) 1512-1533

31. Pijaudier-Cabot, G.; Berthaud, Y.: Damage and interactions in a microcracked medium. Non-local formulation. C. R. Acad. Sci. Paris 310 Série II (1990) 1577-1582

32. Onat, E. T.; Leckie, F. A.: Representation of mechanical behavior in the presence of changing internal structure. Trans. ASME/J, Appl. Mech. 55 (1988) 1-10

33. Onat, E. T.: Effective properties of elastic materials that contain penny-shaped voids. Int. J. Eng. Sci. 22 (1984) 1013-1021

34. Leckie, F. A.; Onat, E. T.: Tensorial nature of damage measuring internal variables. In: Hult, J.; Lemaitre, J. (eds.) Proc. IUTAM Symp. on Physical Nonlinearities in Structures, pp. 140-155. Springer: 1980

35. Evans, A. G.; Marshall, D. B.: The mechanical behavior of ceramic matrix composites. Acta Metall. 37 (1989) 2567-2583

36. Cady, C. M.; Makin, T. J.; Evans, A. G.: J. Am. Ceram. Soc. Silicon Carbide Calcium Aluminosilicate -A Notch-Insensitive Ceramic-Matrix Composite, Vol. 18, No. 1, 77-82, 1995

37. Hutchinson, J. W.; Jensen, H. M.: Models for fiber debonding and fber pullout in brittle composites with friction. Mech. Mat. 41 (1990) 2365

38. Evans, A G.; Domergue, J.-M.; Vagaggini, E.: Methodology for relating the tensile constitutive behavior of ceramic matrix composites to constituent properties. J. Am. Cerarn. Soc. 77 (1994) 1425-1435

39. Allix, O.; Gilletta, D.; Ladeveze, P.: Mechanical behavior of elementary constituents of laminates. Proc. $5^{\text {th }}$ Int. Conf. on Composites Materials (1985) pp. 1039-1057

40. Jansson, S.; Leckie, F. A.: The mechanics of failure of silicon carbide fiber-reinforced glass-matrix composites. Acta Metall. 40 (1993) 2967-2978 
41. Zienkievicz, O. C.; Taylor, R. L.: The finite element method. London: McGraw-Hill 1989

42. Hibbitt, H. D.; Karlsson, B. I.; Sorensen, P.: Abaqus, version 5.3, 1993

43. Chaboche, J.-L.: Le concept de contrainte effective appliquée à l'elasticité et à la viscoplasticité en présence d'un endommagement anisotrope. Colloq. Int. CNRS 295 (1982) 31-43

44. Hild, F.; Burr, A.; Leckie, F. A.: Matrix cracking and debonding in ceramic-matrix composites. accepted for publication in Int. J. Solids Struct.

45. Burr, A.; Hild, F.; Leckie, F. A.: Continuum description of damage in ceramic-matrix composites, University of California, Santa Barbara, U.R.I. Report, 1995

46. Pluvinage, P.: Etude expérimentale et simulation numérique du comportement mécanique de matériaux composites $\mathrm{SiC} / \mathrm{SiC}$. Influence des paramètres de stratification et d'élaboration. Thèse d'Université, 1991 